\title{
Reduction of frequency noise and frequency shift by phase shifting elements in frequency modulation atomic force microscopy.
}

\section{$\operatorname{AUTHOR}(S)$ :}

Kobayashi, Kei; Yamada, Hirofumi; Matsushige, Kazumi

\section{CITATION:}

Kobayashi, Kei ...[et al]. Reduction of frequency noise and frequency shift by phase shifting elements in frequency modulation atomic force microscopy.. The Review of scientific instruments 2011, 82(3): 033702.

\section{ISSUE DATE:}

2011-03

URL:

http://hdl.handle.net/2433/141310

RIGHT:

(C) 2011 American Institute of Physics 


\title{
Reduction of frequency noise and frequency shift by phase shifting elements in frequency modulation atomic force microscopy
}

\author{
Kei Kobayashi, ${ }^{1, a)}$ Hirofumi Yamada, ${ }^{2}$ and Kazumi Matsushige ${ }^{2}$ \\ ${ }^{1}$ Office of Society-Academia Collaboration for Innovation, Kyoto University, Katsura, Nishikyo, \\ Kyoto 615-8520, Japan \\ ${ }^{2}$ Department of Electronic Science and Engineering, Kyoto University, Katsura, Nishikyo, \\ Kyoto 615-8510, Japan
}

(Received 1 January 2011; accepted 31 January 2011; published online 1 March 2011)

\begin{abstract}
We recently reported the analysis of the frequency noise in the frequency modulation atomic force microscopy (FM-AFM) both in high- $Q$ and low- $Q$ environments [Rev. Sci. Instrum. 80, 043708 (2009)]. We showed in the paper that the oscillator noise, the frequency fluctuation of the oscillator, becomes prominent in the modulation frequency lower than $f_{0} / 2 Q$, where $f_{0}$ and $Q$ are the resonance frequency and $Q$-factor. The magnitude of the oscillator noise is determined by the slope of the phase versus frequency curve of the cantilever at $f_{0}$. However, in actual FM-AFM in liquids, the phase versus frequency curve may not be always ideal because of the existence of various phase shifting elements (PSEs). For example, the spurious resonance peaks caused by the acoustic excitation and a band-pass filter in the self-oscillation loop increase the slope of the phase versus frequency curve. Due to those PSEs, the effective $Q$-factor is often increased from the intrinsic $Q$-factor of the cantilever. In this article, the frequency noise in the FM-AFM system with the PSEs in the self-oscillation loop is analyzed to show that the oscillator noise is reduced by the increase of the effective $Q$-factor. It is also shown that the oscillation frequency deviates from the resonance frequency due to the increase of the effective $Q$-factor, thereby causing the reduction in the frequency shift signal with the same factor. Therefore the increase of the effective $Q$-factor does not affect the signal-to-noise ratio in the frequency shift measurement, but it does affect the quantitativeness of the measured force in the FM-AFM. Furthermore, the reduction of the frequency noise and frequency shift by the increase of the effective $Q$-factor were confirmed by the experiments. (O) 2011 American Institute of Physics. [doi:10.1063/1.3557416]
\end{abstract}

\section{INTRODUCTION}

Frequency modulation atomic force microscopy (FMAFM $)^{1-3}$ has been widely used for atomic/molecular-scale investigations of various materials in various environments. In the FM-AFM, the conservative force between the tip and the sample surface is detected as the frequency shift of the cantilever, which is self-oscillated at its resonance frequency. Hence it is important to understand and reduce the frequency noise to achieve high-resolution imaging by the FM-AFM, especially for those operated in low- $Q$ environments, where the quality factor ( $Q$-factor) of the cantilever is extremely damped, such as in liquids.

Recently, we reported the analysis of the frequency noise in the FM-AFM both in high- $Q$ and low- $Q$ environments. ${ }^{4}$ We showed in the paper that the oscillator noise, the frequency fluctuation of the oscillator, contributes to the total frequency noise as well as the frequency measurement noise, which is brought by the measurement of the oscillation frequency. The magnitude of the oscillator noise is determined by the slope of the phase response curve of the cantilever at the resonance frequency $\left(f_{0}\right)$. Since the slope is steep in the frequency range $f_{0} \pm f_{0} / 2 Q$, where $Q$ is the $Q$-factor of the cantilever, the oscillator noise with the modulation frequency $\left(f_{\mathrm{m}}\right)$ lower than $f_{0} / 2 Q$ becomes prominent. In low- $Q$ environments such as in

\footnotetext{
a)Electronic mail: keicoba@iic.kyoto-u.ac.jp.
}

liquids, the corner frequency $\left(f_{\mathrm{c}}\right)$, which is defined as $f_{0} / 2 Q$, becomes very high, and the frequency noise with $f_{\mathrm{m}}$ of concern is governed by the oscillator noise. On the other hand, even though $f_{\mathrm{c}}$ becomes very low in high- $Q$ environments, the oscillator noise becomes dominant when the tip is scanned slowly because $f_{\mathrm{m}}$ of concern becomes low. Therefore understanding the oscillator noise is important not only for the FM-AFM experiments in low- $Q$ environments, but also for those in high- $Q$ environments.

However, it becomes difficult to precisely estimate the oscillator noise in actual FM-AFM experiments because the phase versus frequency curve is often modified by many reasons. First, in many FM-AFM instruments, the cantilever is excited indirectly by the acoustic method, where a piezoelectric actuator is used to vibrate the cantilever base. When the $Q$-factor of the cantilever is low, the actuator excites not only the base of the cantilever, but causes unwanted vibration of surrounding components. ${ }^{5}$ Such spurious resonance peaks of the surrounding components make the identification of the intrinsic resonance frequency of the cantilever difficult by means of measuring the amplitude versus frequency curve and also modify the phase versus frequency curve. In addition, many AFM experimentalists have experienced that the cantilevers do not show clean resonance curves even in air or in vacuum conditions due to nonideal mechanical coupling between the actuator and the cantilever. ${ }^{6}$ Second, a bandpass filter (BPF) or other electronic filters are often used in 
the FM-AFM experiments to eliminate the unwanted noise components other than the resonance frequency range. ${ }^{1}$ It is also useful to avoid the self-oscillation at the spurious resonance peaks mentioned above or at the resonance modes other than the desired mode such as the second eigenmode. Third, when the $Q$-factor of the cantilever is modified by the feedback electronics, ${ }^{7-11}$ the phase versus frequency curve is also altered. It is also reported by several researchers that the $Q$-factor of the cantilever is modified by the use of the FabryPérot interferometer in high- $Q$ environments. ${ }^{12-14}$ Moreover, the phase versus frequency curve might be modified by the limited bandwidth of the displacement sensor electronics, ${ }^{15}$ or by that of the excitation systems. ${ }^{16,17}$ Therefore, the analysis of the frequency noise considering the effect of these phase shifting elements (PSEs), which modify the phase versus frequency curve of the cantilever, is of great importance for the FM-AFM experiments.

In this article we analyze the frequency noise in the FMAFM system with the PSEs in the self-oscillation loop to understand the effect of the PSEs on the frequency noise and the frequency shift signal as well. We first derive the analytical equations for describing the total frequency noise density of the self-oscillated cantilever in the FM-AFM with the PSEs. The analysis is based on an assumption that only the slope of the phase versus frequency curve in the frequency range around $f_{0}$ is modified. Then we derive the oscillation spectrum and corresponding frequency noise density by the numerical analysis so that we can apply the analysis for any FM-AFM systems with the PSEs. We also discuss the effect of the PSEs on the frequency shift signal to discuss the effect of the PSEs on the signal-to-noise ratio of the FM-AFM. Finally, we confirmed the validity of the analysis by the measurement of the frequency noise of the self-oscillating cantilever with a BPF of various $Q$-factors. The effect of the PSEs on the frequency shift signal was also confirmed by the hydration force measurement at the muscovite mica-water interface using both acoustic excitation and photothermal excitation of the cantilever.

\section{DERIVATION OF ANALYTICAL EQUATIONS OF FREQUENCY NOISE DENSITY IN FM-AFM WITH PHASE SHIFTING ELEMENTS IN SELF-OSCILLATION LOOP}

First, we review the analysis of the frequency noise of the self-oscillated cantilever in the FM-AFM, which does not include any PSEs in the self-oscillation loop. ${ }^{4}$ A schematic diagram of the FM-AFM instrument is shown in Fig. 1. We derive the analytical equations of the total frequency noise density as the sum of the oscillator noise and the frequency measurement noise. The oscillator noise, the fluctuation of the oscillator frequency, is determined by the thermal motion of the cantilever displacement noise and the displacement sensor noise within $f_{0} \pm f_{\mathrm{c}}$, while those outside of $f_{0} \pm f_{\mathrm{c}}$ bring additional noise on the frequency measurement, the frequency measurement noise. Since the phase noise of the cantilever turns into the fluctuation of the oscillation frequency by the self-oscillation, the oscillator noise is proportional to the slope of the phase versus frequency curve at the oscillation frequency. The transfer function of the cantilever with the spring constant of $k_{z}$ can be written as

$$
\begin{aligned}
G_{\text {cantilever }}(f) & =\frac{Q}{Q\left(1-\left(f / f_{0}\right)^{2}\right)+j\left(f / f_{0}\right)} \frac{1}{k_{z}} \\
& =\left|G_{\text {cantilever }}(f)\right| \exp [j \theta(f)],
\end{aligned}
$$

where $\theta(f)$ is the phase response of the cantilever to the external force. The frequency noise of the cantilever with the modulation frequency $f_{\mathrm{m}}$ is determined by the thermal displacement of the cantilever $N_{\mathrm{th}}\left(f_{0} \pm f_{\mathrm{m}}\right)$ and the noiseequivalent displacement density (displacement sensor noise) $N_{\mathrm{ds}}\left(f_{0} \pm f_{\mathrm{m}}\right)$. By inserting $f=f_{0} \pm f_{\mathrm{m}}$ to $G_{\text {cantilever }}(f)$, we can approximate $G_{\text {cantilever }}\left(f_{0} \pm f_{\mathrm{m}}\right)$ as

$$
G_{\text {cantilever }}\left(f_{0} \pm f_{\mathrm{m}}\right) \simeq \frac{1}{k_{z}} \frac{-j Q}{1 \pm j\left(f_{\mathrm{m}} / f_{\mathrm{c}}\right)},
$$

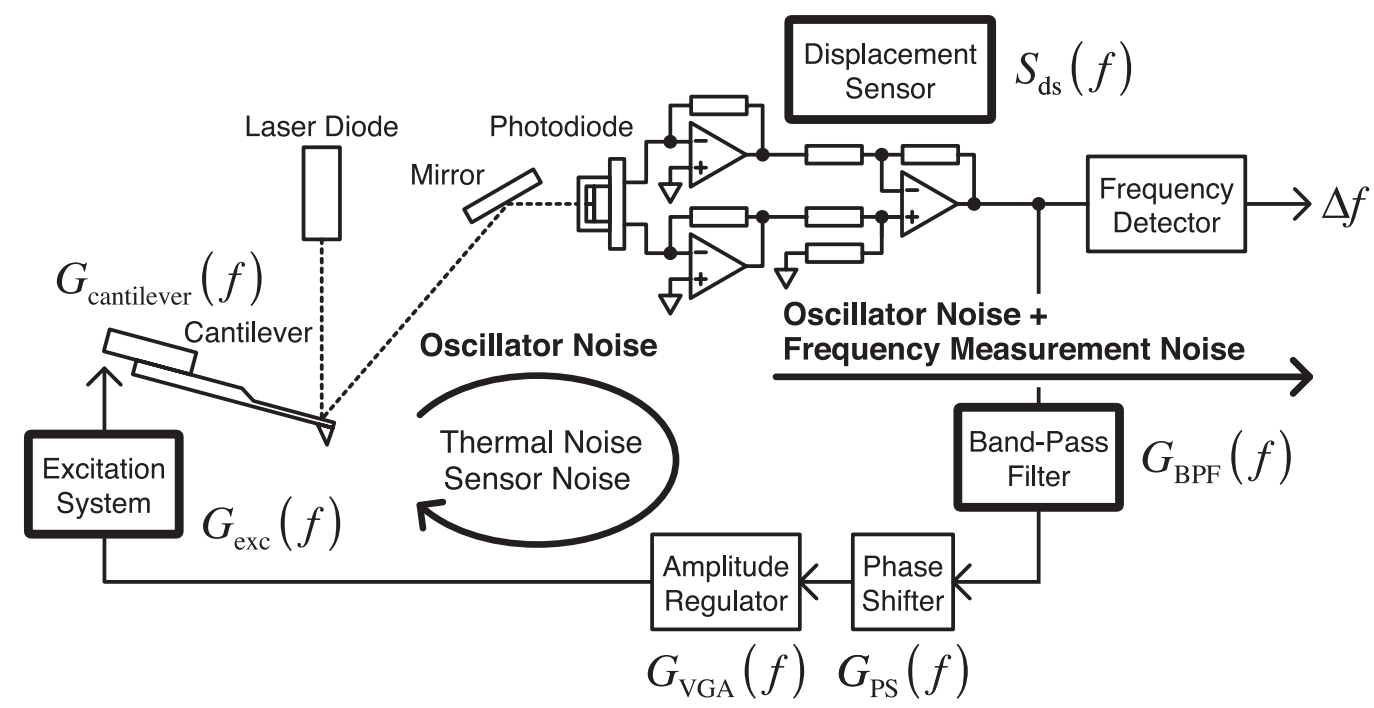

FIG. 1. Schematic of the FM-AFM instrument showing possible sources of the frequency noise. The Brownian motion (thermal displacement noise) of the cantilever and the displacement sensor noise within $f_{0} \pm f_{\mathrm{c}}$, where $f_{\mathrm{c}}$ is $f_{0} / 2 Q$, determine the frequency fluctuation of the oscillator (oscillator noise). The elements shown as thick-line blocks are components which might modify the phase versus frequency shift curve of the cantilever (phase shifting elements). 
within the frequency range $f_{0} \pm f_{\mathrm{c}}$. Therefore $\theta\left(f_{0} \pm f_{\mathrm{m}}\right)$ can be approximated as

$$
\theta\left(f_{0} \pm f_{\mathrm{m}}\right) \simeq-\frac{\pi}{2} \mp \frac{f_{\mathrm{m}}}{f_{\mathrm{c}}}=-\frac{\pi}{2} \mp \frac{2 Q}{f_{0}} f_{\mathrm{m}} .
$$

From this equation, the slope of $\theta(f)$ versus $f$ at $f_{0}$ is described as

$$
\left.\frac{d \theta}{d f}\right|_{f=f_{0}}=-\frac{2 Q}{f_{0}}
$$

which is considered constant within $f_{0} \pm f_{\mathrm{c}}$. From the equipartition theorem, the mean-square displacement of the cantilever end by the thermal energy $k_{\mathrm{B}} T$ is given by

$$
\left\langle z_{\mathrm{th}}{ }^{2}\right\rangle=\int_{0}^{\infty}\left|G_{\text {cantilever }}(f)\right|^{2} F_{\mathrm{th}}{ }^{2} d f=\frac{k_{\mathrm{B}} T}{k_{z}},
$$

where $F_{\mathrm{th}}$ is the magnitude of the random driving force with a white spectral density. Therefore the magnitude of $F_{\text {th }}$ becomes

$$
F_{\mathrm{th}}=\sqrt{\frac{2 k_{z} k_{\mathrm{B}} T}{Q \pi f_{0}}} .
$$

The thermal displacement noise density $N_{\mathrm{th}}\left(f_{0} \pm f_{\mathrm{m}}\right)$ becomes

$$
\begin{aligned}
N_{\text {th }}\left(f_{0} \pm f_{\mathrm{m}}\right) & =\left|G_{\text {cantilever }}\left(f_{0} \pm f_{\mathrm{m}}\right)\right| F_{\mathrm{th}} \\
& =\sqrt{\frac{2 k_{\mathrm{B}} T Q}{\pi k_{z} f_{0}} \frac{1}{1+\left(f_{\mathrm{m}} / f_{c}\right)^{2}}} .
\end{aligned}
$$

Since the displacement noise density at $f_{0}+f_{\mathrm{m}}$ and $f_{0}-f_{\mathrm{m}}$ equally contribute to the frequency noise at the modulation frequency of $f_{\mathrm{m}}$ (See Appendix in Ref. 4), we define $G^{\prime}\left(f_{\mathrm{m}}\right)$, $N_{\mathrm{th}}^{\prime}\left(f_{\mathrm{m}}\right)$, and $N_{\mathrm{ds}}^{\prime}\left(f_{\mathrm{m}}\right)$ which are functions of the modulation frequency for convenience as follows:

$$
\begin{aligned}
& \left|G^{\prime}\left(f_{\mathrm{m}}\right)\right|=\frac{1}{2}\left(\left|G\left(f_{0}+f_{\mathrm{m}}\right)\right|+\left|G\left(f_{0}-f_{\mathrm{m}}\right)\right|\right), \\
& N_{\mathrm{th}}^{\prime}\left(f_{\mathrm{m}}\right)=\frac{1}{2}\left(N_{\mathrm{th}}\left(f_{0}+f_{\mathrm{m}}\right)+N_{\mathrm{th}}\left(f_{0}-f_{\mathrm{m}}\right)\right), \\
& N_{\mathrm{ds}}^{\prime}\left(f_{\mathrm{m}}\right)=\frac{1}{2}\left(N_{\mathrm{ds}}\left(f_{0}+f_{\mathrm{m}}\right)+N_{\mathrm{th}}\left(f_{0}-f_{\mathrm{m}}\right)\right) .
\end{aligned}
$$

Now we assume the displacement sensor noise is constant for the frequency range of concern $\left(f_{0} \pm f_{\mathrm{m}}\right)$ and define $N_{\mathrm{ds}}$ $=N_{\mathrm{ds}}^{\prime}\left(f_{\mathrm{m}}\right)$ for simplicity. Therefore the total displacement noise density including the displacement sensor noise density becomes

$$
\begin{aligned}
N_{\text {total }}^{\prime}\left(f_{\mathrm{m}}\right) & =\sqrt{N_{\mathrm{th}}^{\prime}\left(f_{\mathrm{m}}\right)^{2}+N_{\mathrm{ds}}^{2}} \\
& =\sqrt{\frac{2 k_{\mathrm{B}} T Q}{\pi k_{z} f_{0}} \frac{1}{1+\left(f_{\mathrm{m}} / f_{c}\right)^{2}}+N_{\mathrm{ds}}{ }^{2}} .
\end{aligned}
$$

As discussed in Ref. 4 the oscillator noise is given by

$$
N_{\mathrm{FM}(\mathrm{osc})}\left(f_{\mathrm{m}}\right)=\left|\frac{d f}{d \theta}\right| \frac{\sqrt{2} N_{\mathrm{total}}^{\prime}\left(f_{\mathrm{m}}\right)}{A_{0}}=\frac{f_{0}}{2 Q} \frac{\sqrt{2} N_{\mathrm{total}}^{\prime}\left(f_{\mathrm{m}}\right)}{A_{0}},
$$

thus we obtain

$N_{\mathrm{FM}(\mathrm{osc})}\left(f_{\mathrm{m}}\right)=\sqrt{\frac{f_{0} k_{\mathrm{B}} T}{\pi k_{z} Q A_{0}^{2}} \frac{1}{1+\left(f_{\mathrm{m}} / f_{c}\right)^{2}}+\frac{f_{0}^{2} N_{\mathrm{ds}}{ }^{2}}{2 Q^{2} A_{0}{ }^{2}}}$.

On the other hand, the frequency measurement noise density is given by

$$
N_{\mathrm{FM}(\text { measure })}\left(f_{\mathrm{m}}\right)=\frac{\sqrt{2} N_{\mathrm{total}}^{\prime}\left(f_{\mathrm{m}}\right)}{A_{0}} f_{\mathrm{m}},
$$

and it becomes

$N_{\mathrm{FM}(\text { measure })}\left(f_{\mathrm{m}}\right)=\sqrt{\frac{f_{0} k_{\mathrm{B}} T}{\pi k_{z} Q A_{0}{ }^{2}} \frac{\left(f_{\mathrm{m}} / f_{\mathrm{c}}\right)^{2}}{1+\left(f_{\mathrm{m}} / f_{\mathrm{c}}\right)^{2}}+\frac{2 N_{\mathrm{ds}}{ }^{2}}{A_{0}{ }^{2}} f_{\mathrm{m}}{ }^{2}}$

using Eq. (11). The combination of Eqs. (12) and (15) gives the analytical equation for the frequency noise density in the FM-AFM,

$$
\begin{aligned}
N_{\mathrm{FM}}\left(f_{\mathrm{m}}\right) & =\sqrt{N_{\mathrm{FM}(\mathrm{osc})}{ }^{2}+N_{\mathrm{FM}(\text { measure })}{ }^{2}} \\
& =\sqrt{\frac{f_{0} k_{\mathrm{B}} T}{\pi k_{z} Q A_{0}{ }^{2}}+\frac{f_{0}{ }^{2} N_{\mathrm{ds}}{ }^{2}}{2 Q^{2} A_{0}{ }^{2}}+\frac{2 N_{\mathrm{ds}}{ }^{2}}{A_{0}{ }^{2}} f_{\mathrm{m}}{ }^{2}},
\end{aligned}
$$

which was given as Eq. (18) in Ref. 4. The first term of the equation represents the contribution of the thermal displacement noise, which is constant, and the following terms are those of the displacement sensor noise. The combination of the oscillator noise and the measurement noise to give the total frequency noise is schematically summarized in Fig. 2(a). Note that both axes in the schematics in Fig. 2 are logarithmic. One can obtain the total frequency noise $\delta f$ by integrating the
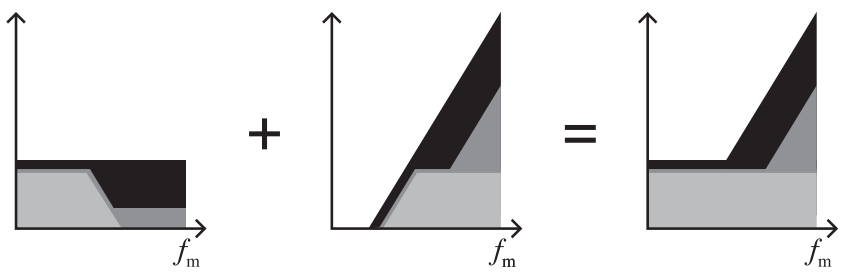

(a)
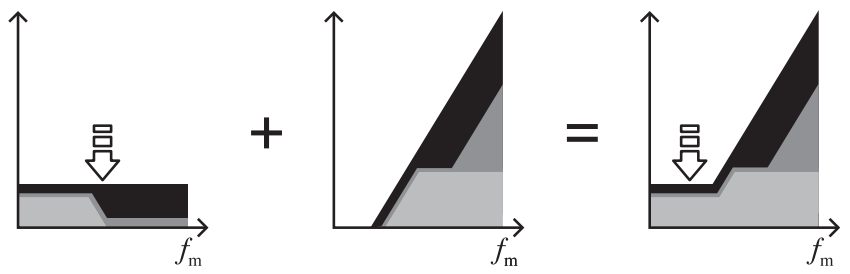

(b)

FIG. 2. (Color online) (a) Schematic of the contribution of the oscillator noise density and the frequency measurement noise density to the total frequency noise density. Dark gray and black areas represent two levels (small and large) of additional displacement sensor noise. (b) Schematic showing the contribution of the oscillator noise, which is reduced by the increase of the effective $Q$-factor, and the frequency measurement noise, which is not affected, to the total frequency noise density in the FM-AFM with the PSE. 
frequency noise density for the bandwidth of $B$ as

$$
\begin{aligned}
\delta f & =\sqrt{\int_{0}^{B} N_{\mathrm{FM}}{ }^{2} d f_{\mathrm{m}}} \\
& =\sqrt{\frac{f_{0} k_{\mathrm{B}} T B}{\pi k_{z} Q A_{0}{ }^{2}}+\frac{f_{0}{ }^{2} N_{\mathrm{ds}}{ }^{2} B}{2 Q^{2} A_{0}{ }^{2}}+\frac{2 N_{\mathrm{ds}}{ }^{2}}{3 A_{0}{ }^{2}} B^{3}} .
\end{aligned}
$$

Now we consider the case when some PSEs such as the BPF are existing in the self-oscillation loop. The displacement sensor or the excitation system which has any frequencydependent phase response in the frequency range of $f_{0} \pm f_{\mathrm{c}}$ is also considered as the PSE. Possible PSEs are indicated in Fig. 1. We assume that the amplitude response of the PSE is considered to be linear within the frequency range of concern $\left(f_{0} \pm f_{\mathrm{c}}\right)$. Due to the PSE, the slope of the effective phase response of the cantilever to the driving force $\left(\theta_{\text {eff }}(f)\right)$ at $f_{0}$ is affected and it is now described as

$$
\left.\frac{d \theta_{\mathrm{eff}}}{d f}\right|_{f=f_{0}}=\left.\frac{d \theta}{d f}\right|_{f=f_{0}}+\left.\frac{d \theta_{\mathrm{add}}}{d f}\right|_{f=f_{0}}
$$

$$
\begin{aligned}
N_{\mathrm{FM}(\mathrm{PSE})}\left(f_{\mathrm{m}}\right) & =\sqrt{\left(\frac{Q_{0}}{Q_{\mathrm{eff}}}\right)^{2} N_{\mathrm{FM}(\mathrm{osc})}{ }^{2}+N_{\mathrm{FM}(\text { measure })}{ }^{2}} \\
& =\sqrt{\frac{f_{0} k_{\mathrm{B}} T}{\pi k_{z} Q_{0} A_{0}{ }^{2}} \frac{\left(Q_{0} / Q_{\mathrm{eff}}\right)^{2}+\left(f_{\mathrm{m}} / f_{\mathrm{c}}\right)^{2}}{1+\left(f_{\mathrm{m}} / f_{\mathrm{c}}\right)^{2}}+\left(\frac{Q_{0}}{Q_{\text {eff }}}\right)^{2} \frac{f_{0}{ }^{2} N_{\mathrm{ds}}{ }^{2}}{2 Q_{0}{ }^{2} A_{0}{ }^{2}}+\frac{2 N_{\mathrm{ds}}{ }^{2}}{A_{0}{ }^{2}} f_{\mathrm{m}}{ }^{2} .}
\end{aligned}
$$

$$
=-\frac{2 Q}{f_{0}}-\frac{2 Q_{\text {add }}}{f_{0}}=-\frac{2 Q_{\text {eff }}}{f_{0}},
$$

where $Q_{\text {eff }}$ is the effective $Q$-factor. $\theta_{\text {add }}$ is the phase transfer function of the PSE, and $Q_{\text {add }}$ corresponds to the $Q$-factor of the PSE, which is defined by the slope of $\theta_{\text {add }}$ at $f_{0}$. Hereafter the intrinsic $Q$-factor of the cantilever is denoted as $Q_{0}$ to avoid confusion. Now the oscillator noise density given by Eq. (12) is modified to

Note that the polarity of $Q_{\text {add }}$ is not necessarily positive depending on the PSE. While the oscillator noise density is decreased by the increase in the effective $Q$-factor, the frequency measurement noise density is not modified from Eq. (15). Thus we obtain the total frequency noise density by the combination of Eqs. (19) and (15) as

$$
\begin{aligned}
N_{\mathrm{FM}(\mathrm{osc}, \mathrm{PSE})}\left(f_{\mathrm{m}}\right) & =\left|\frac{d f}{d \theta_{\mathrm{eff}}}\right| \frac{\sqrt{2} N_{\mathrm{total}}^{\prime}\left(f_{\mathrm{m}}\right)}{A_{0}} \\
& =\frac{f_{0}}{2 Q_{\mathrm{eff}}} \frac{\sqrt{2} N_{\text {total }}^{\prime}\left(f_{\mathrm{m}}\right)}{A_{0}} .
\end{aligned}
$$

The combination of the reduced oscillator noise and the frequency measurement noise to give the total frequency noise in the FM-AFM with PSE is schematically summarized in Fig. 2(b). Figure 3(a) shows the total frequency noise density spectra $N_{\mathrm{FM}(\mathrm{PSE})}\left(f_{\mathrm{m}}\right)$ of a self-oscillated cantilever in liquid $\left(k_{z}=26 \mathrm{~N} / \mathrm{m}, f_{0}=140 \mathrm{kHz}, Q_{0}=9, A_{0}=0.5 \mathrm{~nm}\right)$ measured by the displacement sensor with the noise-equivalent displacement density of $20 \mathrm{fm} / \sqrt{\mathrm{Hz}}$, which were calculated using Eq. (20). Each curve corresponds to the frequency noise density spectrum with the additional $Q$-factor $\left(Q_{\text {add }}\right)$ of 0,1 , $2,5,10$, and 20 . The spectrum for $Q_{\text {add }}=0$ represents the frequency noise density spectrum without any PSE. It should be noted that the total frequency noise density given by Eq. (20) can be approximated by simply multiplying the terms related to the oscillator noise, which are the first and second terms in Eq. (16), with $Q_{0} / Q_{\text {eff }}$ as

$$
N_{\mathrm{FM}(\mathrm{PSE})}\left(f_{\mathrm{m}}\right) \simeq \sqrt{\left(\frac{Q_{0}}{Q_{\text {eff }}}\right)^{2} \frac{f_{0} k_{\mathrm{B}} T}{\pi k_{z} Q_{0} A_{0}{ }^{2}}+\left(\frac{Q_{0}}{Q_{\text {eff }}}\right)^{2} \frac{f_{0}^{2} N_{\mathrm{ds}}{ }^{2}}{2 Q_{0}^{2} A_{0}{ }^{2}}+\frac{2 N_{\mathrm{ds}}{ }^{2}}{A_{0}{ }^{2}} f_{\mathrm{m}}{ }^{2}} .
$$

Figure 3(b) shows $N_{\mathrm{FM}(\mathrm{PSE})}\left(f_{\mathrm{m}}\right)$ calculated using Eq. (21). Parameters used for the calculation are the same as those in Fig. 3(a). The figure shows that the total frequency noise density given by Eq. (21) is essentially the same as that given by Eq. (20) except for the case when $f_{\mathrm{m}}$ is close to $f_{\mathrm{c}}$. 

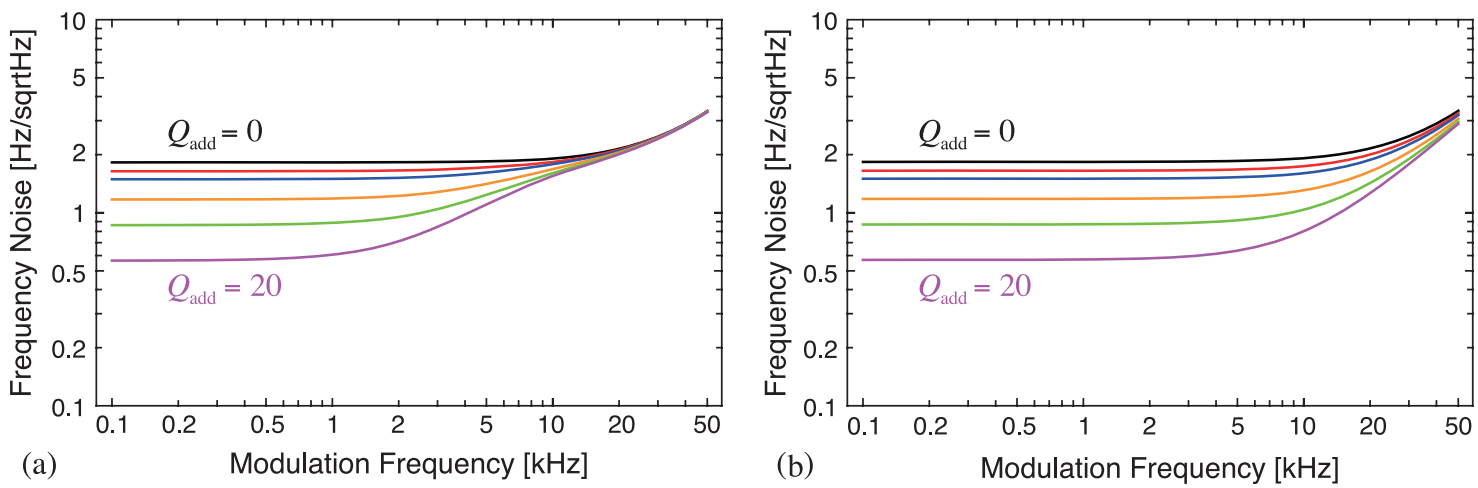

FIG. 3. (Color online) (a) Total frequency noise density spectra $N_{\mathrm{FM}(\mathrm{PSE})}\left(f_{\mathrm{m}}\right)$ of a self-oscillated cantilever in liquid $\left(k_{z}=26 \mathrm{~N} / \mathrm{m}, f_{0}=140 \mathrm{kHz}, Q_{0}=9\right.$, $A_{0}=0.5 \mathrm{~nm}$ ) measured by the displacement sensor with the noise-equivalent displacement density of $20 \mathrm{fm} / \sqrt{\mathrm{Hz}}$, which were calculated using Eq. (20). Each curve corresponds to the spectrum with the additional $Q$-factor $\left(Q_{\text {add }}\right)$ of $0,1,2,5,10$, and 20. (b) Total frequency noise density spectra $N_{\mathrm{FM}(\mathrm{PSE})}\left(f_{\mathrm{m}}\right)$ of a self-oscillated cantilever in liquid calculated using Eq. (21). Parameters used for the calculation are the same as those in (a).

\section{NUMERICAL ANALYSIS OF FREQUENCY NOISE DENSITY IN FM-AFM WITH PHASE SHIFTING ELEMENTS IN SELF-OSCILLATION LOOP}

In Sec. II, we derive the analytical equations of the frequency noise density by assuming the PSE whose amplitude characteristics is flat within the frequency range of concern. The equations give fairly good approximations for cases when $Q_{\text {add }}$ is smaller than $Q_{0}$, but they do not give good approximations any more when $Q_{\text {add }}$ is much larger than $Q_{0}$. Here we describe a method to calculate the displacement density spectrum of the self-oscillated cantilever and then obtain the frequency noise density spectrum. In order to calculate the displacement density spectrum of the self-oscillated cantilever, the transfer function of each element in the self-oscillation loop such as those shown in Fig. 1 has to be determined. For example, if we define the displacement density spectrum of the cantilever and the frequency-dependent displacement sensor sensitivity as $A_{\text {osc }}(f)$ and $S_{\mathrm{ds}}(f)$, respectively, the sensor output signal becomes $S_{\mathrm{ds}}(f) \cdot A_{\mathrm{osc}}(f)$. The transfer functions of the BPF, the phase shifter, and the variable gain amplifier in the amplitude regulator are defined as $G_{\mathrm{BPF}}(f), G_{\mathrm{PS}}(f)$, and $G_{\mathrm{VGA}}(f)$, respectively. Then the excitation signal becomes

$$
V_{\mathrm{exc}}(f)=G_{\mathrm{VGA}}(f) \cdot G_{\mathrm{PS}}(f) \cdot G_{\mathrm{BPF}}(f) \cdot S_{\mathrm{ds}}(f) \cdot A_{\mathrm{osc}}(f) .
$$

As the transfer function of the excitation system $G_{\text {exc }}(f)$, we assume the acoustic excitation using a piezoelectric transducer with a piezoelectric constant of $d_{33}$. Thus the driving force becomes

$$
F_{\text {exc }}(f)=G_{\text {exc }}(f) \cdot V_{\text {exc }}=k_{z} d_{33} V_{\text {exc }}(f) .
$$

Since $A_{\text {osc }}(f)$ is given by the sum of the excited displacement density and the noise-equivalent displacement density as

$$
A_{\text {osc }}(f)=G_{\text {cantilever }}(f) \cdot F_{\text {exc }}(f)+N_{\text {total }}(f),
$$

$A_{\text {osc }}(f)$ becomes

$$
A_{\text {osc }}(f)=\frac{N_{\text {total }}(f)}{1-G_{\text {cantilever }}(f) \cdot k_{z} d_{33} \cdot G_{\mathrm{VGA}}(f) \cdot G_{\mathrm{PS}}(f) \cdot G_{\mathrm{BPF}}(f) \cdot S_{\mathrm{ds}}(f)},
$$

by combining Eqs. (22)-(24). In ideal FM-AFM which we assume, $G_{\mathrm{PS}}(f)$ is set as $G_{\mathrm{PS}}(f)=j$ so that the oscillation frequency matches $f_{0}$. Note that this is not valid when the phase shifter is implemented by an all-pass filter with a variable resistor, which has a frequency-dependent phase response. If we assume $G_{\mathrm{VGA}}(f)$ is constant within the bandwidth of concern, the constant gain $G_{\mathrm{VGA}}$ should become

$$
G_{\mathrm{VGA}}=\frac{1}{G_{\text {cantilever }}\left(f_{0}\right) \cdot k_{z} d_{33} \cdot j \cdot G_{\mathrm{BPF}}\left(f_{0}\right) \cdot S_{\mathrm{ds}}\left(f_{0}\right)},
$$

since the criterion of the self-oscillation requires the total loop gain of 1. By inserting $G_{\mathrm{VGA}}$ to Eq. (25), one can numerically obtain $A_{\text {osc }}(f)$. Once we obtain the displacement density spectrum $A_{\text {osc }}(f)$, the total frequency noise density $N_{\mathrm{FM}(\mathrm{PSE})}\left(f_{\mathrm{m}}\right)$ is obtained as

$$
N_{\mathrm{FM}(\mathrm{PSE})}\left(f_{\mathrm{m}}\right)=\sqrt{\left(A_{\mathrm{osc}}\left(f_{0}+f_{\mathrm{m}}\right)\right)^{2}+\left(A_{\mathrm{osc}}\left(f_{0}-f_{\mathrm{m}}\right)\right)^{2}} \frac{f_{\mathrm{m}}}{A_{0}} .
$$



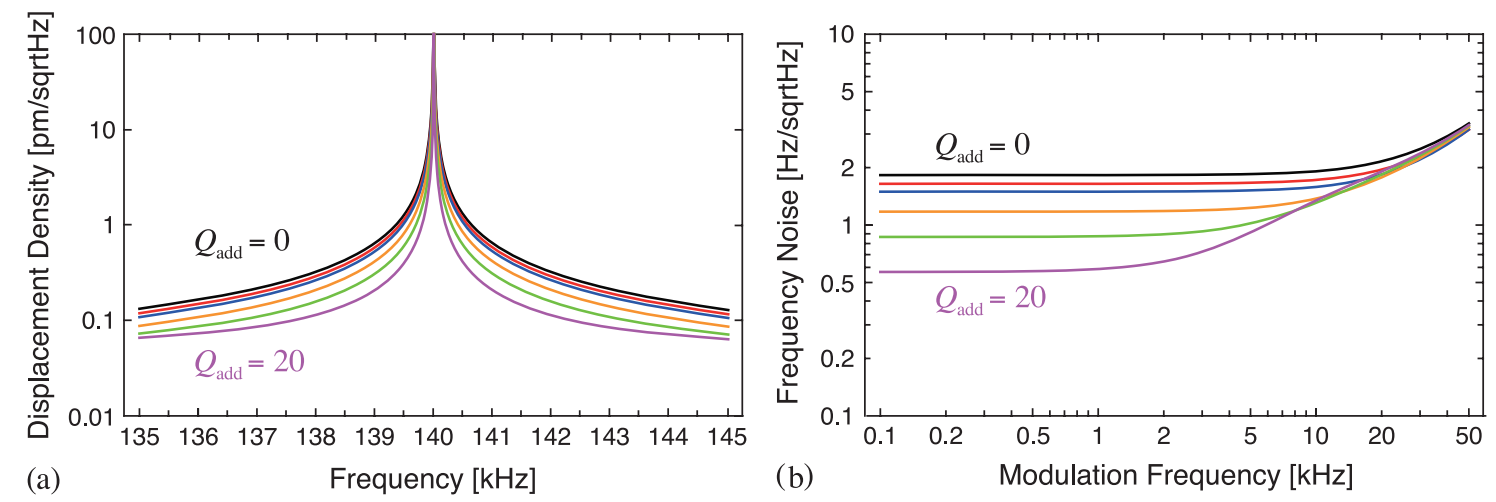

FIG. 4. (Color online) (a) Displacement density spectra $A_{\text {osc }}(f)$ of a self-oscillated cantilever in liquid $\left(k_{z}=26 \mathrm{~N} / \mathrm{m}, f_{0}=140 \mathrm{kHz}, Q_{0}=9, A_{0}=0.5 \mathrm{~nm}\right.$ ) measured by the displacement sensor with the noise-equivalent displacement density of $20 \mathrm{fm} / \sqrt{\mathrm{Hz}}$. Each curve corresponds to the spectrum with $Q_{\text {add }}$ of 0,1 , 2, 5, 10, and 20. (b) Corresponding frequency noise spectra obtained from (a) using Eq. (27).

(See Appendix in Ref. 4). In Fig. 4(a), we show the numerically calculated displacement density spectra $A_{\text {osc }}$ of a self-oscillated cantilever in liquid $\left(k_{z}=26 \mathrm{~N} / \mathrm{m}, f_{0}=140\right.$ $\mathrm{kHz}, Q_{0}=9, A_{0}=0.5 \mathrm{~nm}$ ) measured by the displacement sensor with the noise-equivalent displacement density of 20 $\mathrm{fm} / \sqrt{\mathrm{Hz}}$. We modeled $G_{\mathrm{BPF}}(f)$ as

$$
G_{\mathrm{BPF}}(f)=\frac{j\left(f / f_{0}\right)}{Q_{\mathrm{add}}\left(1-\left(f / f_{0}\right)^{2}\right)+j\left(f / f_{0}\right)},
$$

where $Q_{\text {add }}$ is a quality factor of the $\mathrm{BPF}$ and assumed $S_{\mathrm{ds}}(f)$ is constant. Each curve in Fig. 4(a) corresponds to the spectrum with $Q_{\text {add }}$ of $0,1,2,5,10$, and 20 . Corresponding frequency noise spectra obtained from the displacement density spectra in Fig. 4(a) using Eq. (27) are shown in Fig. 4(b). It is shown by the figures that the linewidth of the oscillator is decreased by increasing the $Q$-factor of the BPF, and the frequency noise density at the lower modulation frequency range is reduced accordingly.

\section{FREQUENCY SHIFT OF FM-AFM WITH PHASE SHIFTING ELEMENTS IN SELF-OSCILLATION LOOP}

In Secs. II and III, we showed that the oscillator noise is reduced by the increase of the effective $Q$-factor of the cantilever by the PSEs in the self-oscillation loop. Here we discuss the effect of the PSEs on the frequency shift signal to discuss the effect of the PSEs on the signal-to-noise ratio of the FM-AFM. The oscillation frequency of the cantilever is the frequency at which the phase criterion

$$
\theta(f)+\theta_{\mathrm{PS}}(f)=2 n \pi
$$

is met, where $\theta(f)$ was given in Eq. (1), and $\theta_{\mathrm{PS}}(f)$ is the phase shift of the phase shifter. Since the phase shift of the cantilever at $f_{0}, \theta\left(f_{0}\right)$, is $-\pi / 2, \theta_{\mathrm{PS}}$ is ideally kept constant at $\pi / 2+2 n \pi$ during experiments. However, due to the presence of the PSE, the phase criterion determining the oscillation frequency in actual FM-AFM experiments becomes

$$
\theta_{\mathrm{eff}}(f)+\theta_{\mathrm{PS}}(f)=2 n \pi .
$$

Schematic of the apparent phase versus frequency curves of the cantilever without any PSE $(\theta(f))$ and with the PSE $\left(\theta_{\text {eff }}(f)\right)$ are shown in Fig. 5(a) as thin and thick curves, respectively. Their slope at $f_{0}$ are given in Eqs. (4) and (18), respectively. Now we consider the situation where $\theta(f)$ is slightly shifted by $\Delta f_{\text {cantilever }}$ due to the tip-sample interaction force, and the resonance frequency moves to $f_{0}+\Delta f_{\text {cantilever }}$. In this situation, the phase response of the cantilever at $f_{0}$ becomes $-\pi / 2+\Delta \theta$, where $\theta$ is given by

$$
\Delta \theta=2 Q_{0} \frac{\Delta f_{\text {cantilever }}}{f_{0}}
$$
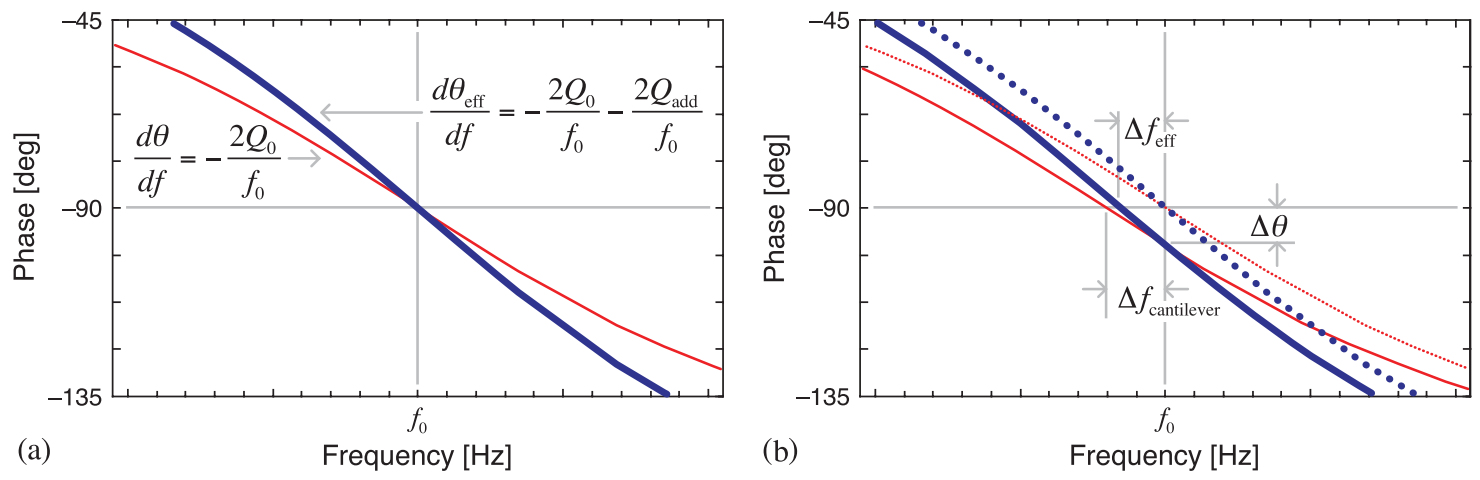

FIG. 5. (Color online) (a) Schematic of apparent phase versus frequency curves of the cantilever without any PSE $\left(\theta(f)\right.$, thin curve) and with the PSE $\left(\theta_{\text {eff }}(f)\right.$, thick curve). The slope of $\theta, d \theta / d f$, is almost constant within the frequency range $f_{0} \pm f_{c}$, and it is increased by the PSE in the self-oscillation loop. (b) Schematic illustration showing that the oscillation frequency deviates from the resonance frequency because of the increase in the effective $Q$-factor by the PSE. 

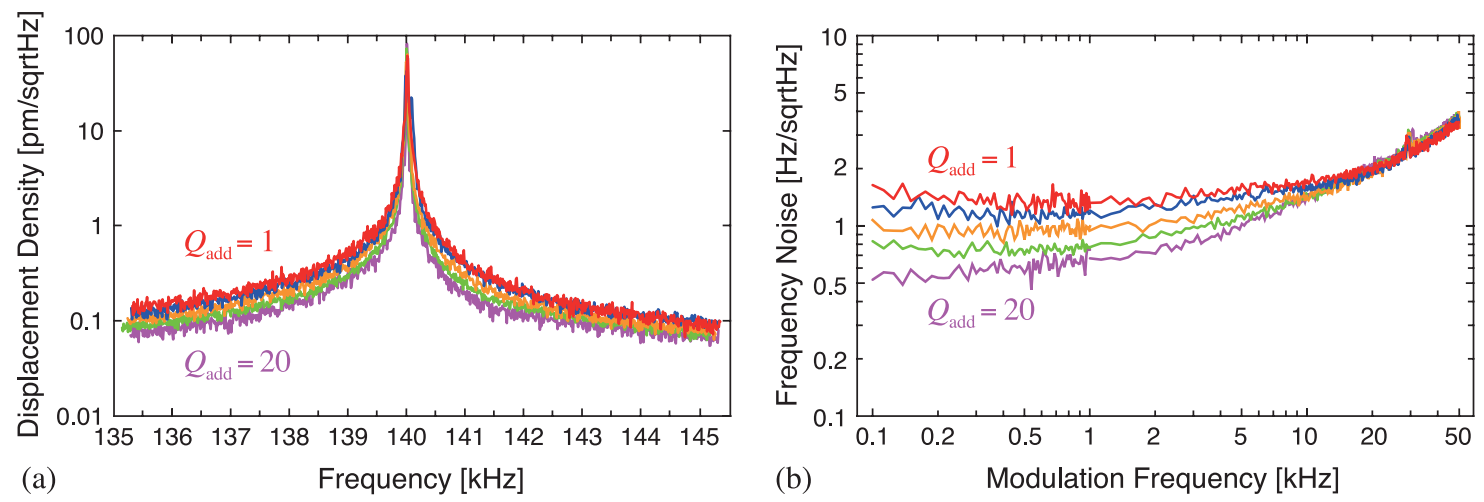

FIG. 6. (Color online) (a) Displacement density spectra of a self-oscillated cantilever in water $\left(k_{z}=26 \mathrm{~N} / \mathrm{m}, f_{0}=140 \mathrm{kHz}, Q_{0}=8.6, A_{0}=0.5 \mathrm{~nm}\right.$ ). Each curve corresponds to the spectrum with $Q_{\text {add }}$ of 1,2,5,10, and 20. (b) Corresponding frequency noise spectra obtained from (a) using Eq. (27).

as shown in Fig. 5(b). This phase shift is instantaneously nulled by the self-oscillation loop, which adjusts the oscillation frequency to $f_{0}+\Delta f_{\text {cantilever. However, with the PSE, the }}$ phase shift $\Delta \theta$ is nulled by adjusting the oscillation frequency to $f_{0}+\Delta f_{\text {eff }}$, which is given by

$$
\Delta f_{\mathrm{eff}}=\Delta \theta\left(\left|\frac{d \theta_{\mathrm{eff}}}{d f}\right|_{f=f_{0}}\right)^{-1}=\frac{Q_{0}}{Q_{\mathrm{eff}}} \Delta f
$$

where $\Delta f_{\text {eff }}$ represents the effective frequency shift tracked by the self-oscillation loop with the PSE. If we compare Eqs. (20) or (21) with Eq. (32), both the signal and noise decreases by the same factor of $Q_{\text {eff }} / Q_{0}$, therefore, the signal to noise ratio of the FM-AFM is eventually not affected by the increase of the effective $Q$-factor.

\section{MEASUREMENT OF FREQUENCY NOISE WITH PHASE DELAY ELEMENTS IN SELF-OSCILLATION LOOP}

We measured the frequency noise of a self-oscillated cantilever in liquid to show the validity of the discussion on the effect of the PSEs on the frequency noise. We used a highly doped n-type Si cantilever with gold reflex coating on the backside (Nanosensors: NCH-AuD), whose spring constant was $26 \mathrm{~N} / \mathrm{m}$, calibrated using Sader's method in air. ${ }^{18}$ The cantilever was immersed in water and the resonance frequency and $Q$-factor in water were $140 \mathrm{kHz}$ and 8.6, respectively. We used a commercial AFM head (Shimadzu: SPM9500) after some modifications to the optics and electronics to reduce the displacement sensor noise in the optical beam deflection sensor as described in Ref. 15. The thermal displacement density spectrum was measured in water, and it was fitted to the simple harmonic oscillator model. ${ }^{19}$ The sensitivity of the displacement sensor was calibrated, and the displacement sensor noise density was determined as about $23 \mathrm{fm} / \sqrt{\mathrm{Hz}}$. A band-pass filter implemented using a statevariable filter module (NF Corporation: DT-208D), whose center frequency was set at $140 \mathrm{kHz}$, was inserted in the selfoscillation loop as shown in Fig. 1. We used home-built selfoscillation electronics with a phase shifter (NF Corporation: CD-951V4). ${ }^{20}$ The cantilever was excited by an intensitymodulated blue-violet laser (Sanyo: DL-LS5042) driven by a laser-diode driver (Wavelength Technology: WLD3343) to avoid exciting at the spurious resonance peaks. ${ }^{21,22}$ The cantilever was self-excited at $140 \mathrm{kHz}$ with an oscillation amplitude of $1.0 \mathrm{~nm}$ peak-to-peak. We varied $Q_{\text {add }}$ from 1 to 20, and measured the frequency spectra of the displacement signal using a spectrum analyzer (Agilent Technologies: 4395A). Since the demodulation bandwidth of the frequency detector was limited at about $10 \mathrm{kHz},{ }^{20}$ we calculated the frequency noise density spectra from the measured displacement density spectra.

Figure 6(a) shows the displacement density spectra of the self-oscillated cantilever in water $\left(k_{z}=26 \mathrm{~N} / \mathrm{m}, f_{0}=\right.$ $\left.140 \mathrm{kHz}, Q_{0}=8.6, A_{0}=0.5 \mathrm{~nm}\right)$. Each curve corresponds to the spectrum with $Q_{\mathrm{BPF}}$ of $1,2,5,10$, and 20 . Corresponding frequency noise spectra obtained from Fig. 6(a) using Eq. (27) are shown in Fig. 6(b). The frequency noise density spectra for the modulation frequency lower than 1 $\mathrm{kHz}$ were calculated from the displacement density spectra measured with the frequency span of $f_{0} \pm 1 \mathrm{kHz}$ (not shown). Figures 6(a) and 6(b) are quantitatively consistent to Figs. 4(a) and 4(b), respectively. Possible reasons of slight difference between the theoretical spectra and the measured spectra are deviation of the cantilever characteristics from the simple harmonic oscillator model given in Eq. (1), ${ }^{23}$ errors in the parameters used for the calculation and nonideal circuit characteristics.

\section{MEASUREMENT OF FREQUENCY SHIFT WITH PHASE DELAY ELEMENTS IN SELF-OSCILLATION LOOP}

We performed hydration force measurements on mica to show the validity of the discussion on the effect of the PSEs on the frequency shift. The experimental setup for the measurement was the same as described in Sec. V. A freshly cleaved muscovite mica substrate was immersed in $1 \mathrm{~mol} / \mathrm{l}$ $\mathrm{KCl}$ aqueous solution. First, the cantilever was excited by a piezoelectric actuator attached close to the cantilever. We measured the two-dimensional frequency shift map as described in Ref. 24 and frequency shift curves averaged for ten consecutively measured frequency shift curves is plotted at the top of Fig. 7(a). Then we performed the same experiments by the photothermal actuation of the cantilever. 

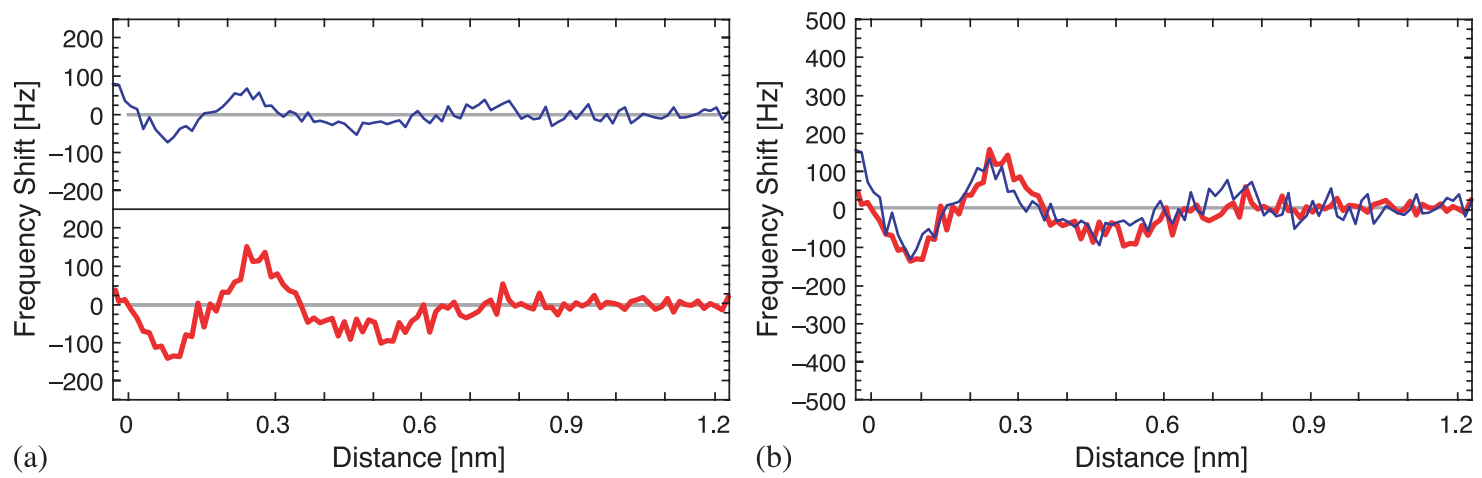

FIG. 7. (Color online) (a) Averaged frequency shift curves measured in $1 \mathrm{~mol} / \mathrm{KCl}$ solution using acoustic actuation (top) and photothermal excitation (bottom). (b) Averaged frequency shift curves in (a) plotted together after correction for the curve acquired with the acoustic actuation using Eq. (33).

The frequency shift curves averaged for ten consecutively measured frequency shift curves is plotted at the bottom of Fig. 7(a).

The frequency shift variation due to the hydration force is smaller in the frequency shift curve measured by the acoustic actuation. We measured the frequency response of the cantilever from the excitation signal to the displacement signal, which is equivalent to $G_{\mathrm{BPF}} \cdot S_{\mathrm{ds}} \cdot G_{\text {cantilever }} \cdot G_{\text {exc }}$ in Fig. 1, from which we calculated the effective $Q$-factor. It was about 16 , mainly due to the spurious resonance peaks of the fluid cell. The resonance frequency shift can be corrected by the equation,

$$
\Delta f=\frac{Q_{\text {eff }}}{Q_{0}} \Delta f_{\text {measured }}
$$

derived from Eq. (32), where $\Delta f_{\text {measured }}$ is the measured frequency shift. The curve acquired with the acoustic actuation was corrected using Eq. (33). Figure 7(b) shows averaged frequency shift curves measured in $1 \mathrm{~mol} / \mathrm{l} \mathrm{KCl}$ solution plotted together after correction for the curve acquired with the acoustic actuation. They are quantitatively coinciding with each other, which proves that the discussion on the effect of the PSEs on the frequency shift is valid. The frequency shift measured with the FM-AFM system including PSEs in the self-oscillation loop could be under/overestimated by the increase/decrease in the effective $Q$-factor.

\section{CONCLUSIONS}

We analyzed the frequency noise of the cantilever in FMAFM considering the frequency-dependent phase shifting elements in the self-oscillation loop. We first derived the analytical equations for describing the total frequency noise density of the self-oscillated cantilever in the FM-AFM with the PSEs and showed that the oscillator noise decreases with increasing effective $Q$-factor. Then we presented numerical calculation of the frequency noise density spectrum of the self-oscillated cantilever, which can be applied for the self-oscillation loop including general PSEs. We also showed that the frequency shift is no longer the same as the resonance frequency if the PSE exists in the self-oscillation loop. The frequency shift decreases with the same factor with the decrease in the oscillator noise by the increase of the effective $Q$-factor by the PSEs.
Even though the signal-to-noise ratio in the frequency measurement in the FM-AFM is not affected by the increase of the effective $Q$-factor, it does affect the quantitativeness of the measured force in the FM-AFM. The reduction of the frequency noise and frequency shift signal by the increase of the effective $Q$-factor were confirmed experimentally by the frequency noise measurements and the hydration force measurements on mica, and the frequency shift versus distance curve measured by the acoustic excitation was corrected. One has to take a great care for quantitative force measurement with the FM-AFM if the PSE could exist in the self-oscillation loop independent of the operating environments.

\section{ACKNOWLEDGMENTS}

This work was supported by Grant-in-Aids for Scientific Research from the Ministry of Education, Culture, Sports, Science, and Technology of Japan, SENTAN Program of the Japan Science and Technology Agency, and Global COE Program of the Japanese Society for the Promotion of Science.

${ }^{1}$ T. R. Albrecht, P. Grütter, P. Horne, and D. Rugar, J. Appl. Phys. 69, 668 (1991).

${ }^{2}$ Noncontact Atomic Force Microscopy, edited by S. Morita, R. Wiesendanger, and E. Meyer (Springer, Berlin, 2002) and references therein.

${ }^{3}$ Noncontact Atomic Force Microscopy, edited by S. Morita, F. J. Giessibl, and R. Wiesendanger (Springer, Berlin, 2009), Vol. 2 and references therein.

${ }^{4}$ K. Kobayashi, H. Yamada, and K. Matsushige, Rev. Sci. Instrum. 80, 043708 (2009).

${ }^{5}$ T. Schäffer, J. P. Cleveland, F. Ohnesorge, D. A. Walters, and P. K. Hansma, J. Appl. Phys. 80, 3622 (1996).

${ }^{6}$ J. D. Adams, D. York, and N. Whisman, Rev. Sci. Instrum. 75, 2903 (2004).

${ }^{7}$ J. Mertz, O. Marti, and J. Mlynek, Appl. Phys. Lett. 62, 2344 (1993).

${ }^{8}$ K. J. Bruland, J. L. Garbini, W. M. Dougherty, and J. A. Sidles, J. Appl. Phys. 80, 1959 (1996).

${ }^{9}$ B. Anczykowski, J. P. Cleveland, D. Krger, V. Elings, and H. Fuchs, Appl. Phys. A 66, S885 (1998)

${ }^{10}$ A. D. L. Humphris, J. Tamayo, and M. J. Miles, Langmuir 16, 7891 (2000).

${ }^{11}$ D. Ebeling, H. Hölscher, and B. Anczykowski, Appl. Phys. Lett. 89, 203511 (2006).

${ }^{12}$ C. Höhberger and K. Karrai, Nature (London) 432, 1002 (2004).

${ }^{13}$ C. Metzger, I. Favero, A. Ortlieb, and K. Karrai, Phys. Rev. B 78, 035309 (2008). 
${ }^{14}$ H. Hölscher, P. Milde, U. Zerweck, L. M. Eng, and R. Hoffmann, Appl. Phys. Lett. 94, 223514 (2009).

${ }^{15}$ T. Fukuma, M. Kimura, K. Kobayashi, K. Matsushige, and H. Yamada, Rev. Sci. Instrum. 76, 053704 (2005).

${ }^{16}$ M. Kageshima, T. Chikamoto, T. Ogawa, Y. Hirata, T. Inoue, Y. Naitoh, Y. J. Li, and Y. Sugawara, Rev. Sci. Instrum. 80, 023705 (2009).

${ }^{17}$ V. Pini, B. Tiribilli, C. M. C. Gambi, M. Vassalli, Phys. Rev. B 81, 054302 (2010).

${ }^{18}$ J. E. Sader, J. W. M. Chon, and P. Mulvaney, Rev. Sci. Instrum. 70, 3967 (1999).
${ }^{19}$ J. L. Hutter and J. Bechhoefer, Rev. Sci. Instrum. 64, 1868 (1993).

${ }^{20}$ K. Kobayashi, H. Yamada, H. Itoh, T. Horiuchi, and K. Matsushige, Rev. Sci. Instrum. 72, 4383 (2001).

${ }^{21}$ N. Umeda, S. Ishizaki, and H. Uwai, J. Vac. Sci. Technol. B 9, 1318 (1991).

${ }^{22}$ G. C. Ratcliff, D. A. Erie, and R. Superfine, Appl. Phys. Lett. 72, 1911 (1998).

${ }^{23}$ J. E. Sader, J. Appl. Phys. 84, 64 (1998).

${ }^{24}$ K. Kimura, S. Ido, N. Oyabu, K. Kobayashi, Y. Hirata, T. Imai, and H. Yamada, J. Chem. Phys. 132, 194705 (2010). 